\title{
AI: The Missing Link in Digital Game Interface Design?
}

\author{
Darryl Charles ${ }^{1}$ and Daniel Livingstone ${ }^{2}$ \\ ${ }^{1}$ Computing and Information Engineering, University of Ulster, \\ BT52 1SA. Northern Ireland \\ dk.charles@ulster.ac.uk \\ ${ }^{2}$ School of Computing, University of Paisley, \\ Paisley, PA12BE. Scotland \\ daniel.livingstone@paisley.ac.uk
}

\begin{abstract}
The central problem that this paper addresses is how to manage dynamic change within game environments in response to variable player requirements and ability. In particular, we discuss the role of a game AI to enable game systems to learn about individual user patterns, behaviours, desires or moods in order to adapt the environment in reaction to the user's interaction with the environment. We discuss the role that AI may play in the design of the game interface in order to enhance the dynamic and responsive nature of the game system with respect to individual users and leading to more rewarding and immersive game experiences.
\end{abstract}

\section{Introduction}

There are many challenges for the use of artificial intelligence (AI) in next generation games [3], but as the power of computers and game consoles advance rapidly there are also many opportunities. In this paper we explore the role of AI within a game as facilitator for the gaming processes - the central goal being to intelligently enhance the interactive experience for individual players. While the concept of an adaptive game is a controversial topic among some gamers and developers, there are clear benefits to tailoring the game experience to particular player types - especially for educational games [2].

\section{AI as an Interface Between Gamer and Game Environment}

We propose that there are three aspects to an intelligent game interface that must be implemented so that a game may be effectively sensitive to player type. These are: modelling the player or player groups, recognising the player in order to adapt the game, and the verification that any adaptation is successful or appropriate.

Current games do little to automatically adapt to individual players needs or desires. However, it is a common feature of games to allow the player to adapt the game themselves; from simple examples like adjusting the difficulty level in games such as "Doom" and the tailoring of a character to a player's requirements at the start 
of a game as with "DeusEx", through to the player sculpting of a game world as happens in "The Sims".

Game developers have been most effective and imaginative in designing methods for dynamic assistance to a player - though examples are still relatively rare and most methods are far from providing intelligent support. One example that can be very effective is simply to track a player's progress and provide help to a player if he or she meets difficulty in a game. A straightforward scheme for this is demonstrated in "Crash Bandicoot". If the player repeatedly fails at a particular point, their character is awarded a magic mask - then no matter how badly the player does on the next attempt, their character will successfully negotiate the offending obstacle. This help is generally accepted willingly by the player; however this is not always the case, for example in "Mario Kart" the game provides a player who is losing the race with the most effective power-ups - which often frustrates their opponent who is normally disadvantaged by this positive discrimination.

Detecting when a player is having difficulty in a less linear games is often not as simple and may require more intelligent methods for understanding and detecting a player's difficulty. More advanced techniques may require the modelling of typical players and behaviours and mapping these to desired game responses, so that in-game we may track player patterns, identify behaviours and execute predefined actions. An example of where this might be useful is in a squad based game where players are able to ask other characters to carry out tasks. With an intelligent system analyzing the players' actions and intent, squad members may be able to pro-actively offer to carry out tasks. This would lead to less need for the player to micro-manage other characters - some element of control will be reduced to agreeing or disagreeing to offers of help. If implemented well it would also increase the perceived intelligence of the computer controlled squad members, and increasing the degree of immersion in the game overall.

\section{Learning to Adapt to Individual Players}

There are still relatively few well known games that use learning algorithms either in development or, even more rarely, within the game. Examples include "Colin McRae Rally 2" which uses neural networks to train to the non-player vehicles to drives realistically on the track, and "Black \& White" which uses neural learning processes within modules to model an avatar's desire. Learning will become more important in next generation games because it will enable a more tailored experienced for individual game players and so we explore some of the issues related to learning.

Learning mechanisms may be offline or online. With offline learning we train the AI during the development process by forcing it to observe and model player behaviour [4] using learning algorithms such as artificial neural networks [6]. This may be used to create believable characters by imitation of a typical (or expert) player or combination of features from a variety of players, or perhaps offline learning may be used to model players in order to respond appropriately a player in-game. Online learning is a much more difficult prospect because it is a real-time process and many of the commonly used algorithms for learning are therefore not suitable. 
There are two important aspects of learning that need to be considered within a game AI interface that is responsive to a player: that of the player learning to use the game system to its full potential and/or according to their needs, and that of the system learning to react to individual players' needs and desires. Both forms of learning are intrinsically linked in a game, i.e. by learning about the player then the game system should be more empowered in helping the player to play the game effectively. Intelligent methods may be built into the game to recognise player characteristics based on prior training performed during the game development and the game may continue to learn on the basis of new information.

However, adapting the environment based on our "belief" about the needs of the player is dangerous - if the change is very obvious or if it frequently disadvantages the player, then it may not be a popular technology. There are two opposing desires in players that we need to take into account: the desire of a player to learn the rules so as to master the game, and the requirement to avoid "sameness" or lack of variety of gameplay.

Even if we achieve this balance there are other issues due to the many difficulties in dealing with dynamic AI behaviours. The most significant issue with the implementation of this type of technology being that on-line learning can often produce very unpredictable results; sometimes these effects serve to enhance but more often it leads to erratic behaviour that may reduce the quality of experience for the user, and in worse scenarios will introduce dynamic software bugs [1].

\section{How and What to Learn About the Player?}

Knowing how and what to learn about a player is just as difficult as actually implementing the technology to perform the learning. One method that we may use to create typical player profiles is to cluster similar types of player together on the basis of player selected preferences and observing players during the game's development. We can then use these typical player groups to make decisions in-game that tailor the game experience better to an individual player. It is also possible for the game to learn how to react appropriately to a player dynamically as the game progresses. However, the question is: which aspects of the players' interaction with the game should we observe and when should the information gained be used? The information that the game has about a player may unambiguously point to a certain course of action but an incorrect transition in the game environment state may still be the result if we do not take into account player intension, desire or mood. These are difficult things gauge but if we were able to extract accurate information then it could vastly improve the performance of game state transition in response to player actions or behaviour.

Monitoring players' actions through input devices, such a mouse, keyboard or game pad is relatively easy. We can also track a players' progress, pattern of play and choices made, and all of this information may be used to form quite a clear picture of the player type. Using pre-constructed player profiles we can correlate the player patterns and make decisions on that basis. Using a pre-trained classifier within the game to identify player patterns can be very fast and efficient. However, these methods are not very good, by-in-large, for dynamic or adaptive learning purposes 
and a further difficulty is that player profiles ideally need to be adapted dynamically because a player's ability and way of playing will change as they play.

We may make even more accurate choices about the player state and therefore more informed decisions about the adaptation of the game environment in response to the players' needs if we gain information or at least clues about player intention, desire or mood. Devices similar to the EyeToy on the PlayStation 2 or a Web Cam on a PC may be used in the future to detect gestures and facial expressions that may provide valuable clues about a persons' mood. Similarly, the pressure exerted by pressing analog game pad buttons may provide information about a players' game style or current level of intensity or stress. Additionally, with simple modification of existing input devices temperature or pulse (i.e. heart rate) sensors may be added like those on a typical exercise bicycle.

Armed with information from standard sources such as the mouse or joy pad and more advanced sources such as a camera or other sensory devices, we have a wealth of information to make decisions that tailor the game to individual players.

\section{Conclusion}

Modern digital games are extraordinarily good at many things but even the best examples of these games are still not very capable at monitoring players, distinguishing between different player groups and altering the game state to meet individual players' needs. In this paper we have outlined the role that AI may play in the interface between player and game so as to respond to individual players and thus improve a players' game playing experience. The improvements that this may bring can be as straightforward as helping the player learning how to play the game or to react when the player becomes "stuck", through to encouraging gameplay innovation in digital games.

\section{References}

1. Barnes $\mathrm{J}$ and Hutchens $\mathrm{J}$, "Testing Undefined Behaviour as a Result of Learning", AI Game Programming Wisdom, pp 615-623, Charles River Media, 2002.

2. Beal, C., Beck, J., Westbrook, D., Atkin, M., and Cohen, P., 2002, Intelligent Modeling of the User in Interactive Entertainment. In AAAI Spring Symposium on Artificial Intelligence and Interactive Entertainment. Stanford, CA.

3. Charles D, "Enhancing Gameplay: Challenges for Artificial Intelligence in Digital Games", Proceedings of the 1st World Conference on Digital Games 2003, University of Utrecht, The Netherlands, 4-6th November 2003.

4. Houlette R, "Player Modelling for Adaptive Games", pp 557-566, AI Game Programming Wisdom II, Charles River Media, 2004.

5. Livingstone D. \& Charles D, "Intelligent Interfaces for Digital Games", AAAI-04 Workshop on Challenges in Game AI, (to be presented) 25-26 $6^{\text {th }}$ July 2004.

6. McGlinchey S, "Learning of AI Players from Game Observation Data", GAME-ON 2003, $4^{\text {th }}$ International Conference on Intelligent Games and Simulation, pp. 106-110, Nov. 2003. 